\title{
Research Paper \\ Comparing the Pattern of Lower Limb Joints Coordination in an Optional and Selective Sprint Start of Elite Women Runners
}

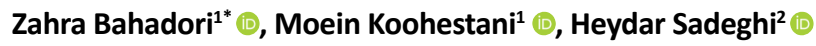 \\ 1. Department of Sport Biomechanics, Faculty of Physical Education and Sport Sciences, Kharazmi University, Tehran, Iran. \\ 2. Department of Sports Biomechanics, Institute of Motor Sciences, Kharazmi University, Tehran, Iran.
}

\begin{tabular}{l|l}
$\begin{array}{c}\text { use your device to san } \\
\text { and read the article online }\end{array}$ & $\begin{array}{l}\text { Citation: Bahadori Z, Koohestani M, Sadeghi H. [Investigating and Comparing the Pattern of Lower Limb Joints Coordination } \\
\text { in an Optional and Selective Sprint Start of Elite Women Runners (Persian)]. Journal of Sport Biomechanics. 2021; 6(4):276-289. } \\
\text { https://doi.org/10.32598/biomechanics.6.3.6 }\end{array}$ \\
dofihttps://doi.org/10.32598/biomechanics.6.3.6
\end{tabular}

(c) (i) (5)

Article Info:

Received: 16 Nov 2020

Accepted: 21 Nov 2020

Available Online: 01 Mar 202

Keywords:

Sprint start, Lower

limbs coordination,

Continuous relative

phase, Elite runner

\section{A B S TRACT}

Objective The sprint start is a complex skill characterized by a multi-joint and multi-plane task requiring complex muscle coordination to reach a large force exerted in the horizontal direction in a short time. Previous studies indicated that efficient acceleration over the first portion of a race is influenced by how a sprinter is positioned in the set command blocks.

Methods A total of 15 elite women runners participated in this study. The subjects performed three optional, and five selected sprint starts with 2-minute intervals. The Noraxon-MyoMotion device collected the kinematic data, and a continuous relative phase method was used to calculate the joint coordination pattern

Results The pattern of coordination between the lower limb joints were divided into 10 phases. There were differences between the two types of starters in the initial phases, but no difference was noticed from the fourth phase.

Conclusion Indeed, there was irregularity in the early phases of the selected start type, while in the following phases, the coordination pattern coincided, and it seems that if this process does not affect the speed and acceleration of the athlete, it can be cautiously noted that sitting in any way in the start technique will ultimately create common coordination in the joints.

\section{Extended Abstract}

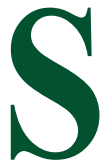

\section{Introduction}

print running is an integral part of most sports [1]. A quick start is an important skill for the runner to have a maximum performance during the race. The initial phase is a complex skill that requires complex muscular coordination with several joints and in different movement planes to reach a large forward force in the shortest time
[2]. Achieving adequate acceleration in the first part of a sprint is influenced by how the runner is positioned on the blocks and the mechanism of detachment from the block at the moment of gunshot [3]. The kinetic and kinematic patterns of elite athletes in the start and acceleration phases have received much attention in recent studies [4-6].

These studies' results show an essential component of the starting technique is the geometry and the body's position when sitting in the starting position including the block's position, the center of mass, and the body's

\section{* Corresponding Author:}

Zahra Bahadori, MA.

Address: Department of Sport Biomechanics, Faculty of Physical Education and Sport Sciences, Kharazmi University, Tehran, Iran.

Tel: +98 (935) 6403920

E-mail: zh.bahadori1991@gmail.com 
angle. The optimal angle of the front and back legs in the posture position is the primary determinant of body shape to achieve a fast horizontal speed at the moment of detachment [8, 7]. However, accurate information about the initial phase and the first steps of running can be important for coaches to improve and develop their understanding of these two stages' movements. This study aimed to compare the lower limb kinematic parameters of elite female runners in two different types of starts. This analysis shows the coordination between the joints of the limb in the start phase.

\section{Method}

This study's statistical population was women runners, 15 to 25 years old in Tehran city. From the statistical population, 15 people were participated by random sampling available method with a Mean \pm SD age of $3.57 \pm 17.93$ years, Mean \pm SD weight $5 \pm 66.5 \mathrm{~kg}, \mathrm{Mean} \pm \mathrm{SD}$ height $166.26 \pm 4.99 \mathrm{~cm}$, and a 100-meter running record of $13.88 \pm 0.33$ seconds.

The Noraxon-MyoMotion device made in the USA was used to measure the kinematic variables in the starting skill. Each subject warmed up for 10 minutes, and after placing the sensors, they each made their start, which they do in competitions, 3 times every 2 minutes. Then each subject made a long start 5 times every 2 minutes. The Continuous Relative Phase (CRP) method was used due to the importance of movement speed in starting skills [17-19], in which a phase angle is obtained after obtaining the location of the normalized angle and the normalized angular velocity.

To analyze the start skill data, all coordination data were normalized to $100 \%$ (from the first move of the subject to the first step after the start line) [21-23]. The Continuous Relative Phase (CRP) of the hip and knee joint, thigh and ankle, knee and ankle during the start movement was calculated [17-19]. The most common values derived from CRP data include the average over a separate period of the cycle, with angles averaging every $10 \%$ of the cycle [12]. The normalized data in this study were also averaged during every $10 \%$ of the cycle.

\section{Results}

The lower limb coordination pattern in the subjects was calculated and was drawn in graphs in 100 pieces. Then, for statistical calculations in every $10 \%$ of the cycle, averaging was performed, and the parts where the difference between the two starts was significant were listed.
Results in knee-to-ankle coordination pattern: first phase: $(\mathrm{P}=0.021)$, second phase: $(\mathrm{P}=0.03)$; thigh-toknee coordination pattern: second phase $=(\mathrm{P}=0.025)$, third phase $=(\mathrm{P}=0.041)$, fourth phase $=(\mathrm{P}=0.018)$, fifth phase $=(\mathrm{P}=0.01)$; thigh-back knee coordination pattern: first phase $=(\mathrm{P}=0.035)$, second phase $=(\mathrm{P}=012)$, and third phase $=(\mathrm{P}=0.008)$; thigh-to-ankle coordination pattern: ninth phase $=(\mathrm{P}=0.018)$; thigh-to-ankle coordination pattern: first phase $=(\mathrm{P}=0.01)$, second phase $=(\mathrm{P}=0.002)$, and seventh phase $=(\mathrm{P}=0.06)$ showed a significant difference and in other cases no significant difference was observed.

\section{Discussion and Conclusion}

This study showed that the initial phases' lower limb coordination pattern was significantly different between the two types of starters, but no difference was noticed from the fourth phase. In general, in coordination diagrams, when CRP is equal to 0 degrees, the movement of the two oscillators is in a phase that is, the two oscillators move in the same direction, and the CRP angle of 180 degrees indicates a completely anti-phase of the two oscillators, they also show an opposite movement oscillator. Each CRP angle between 0 and 180 degrees indicates out-of-phase fluctuations that can be either in-phase or out of phase. Positive CRP values indicate that the distal limb is advanced in phase space, and negative CRP indicates proximal limb proximity. Scott stated that a quick start, higher speed, and long start cause the person to push forward more. Also, the running analysis shows that a long start is associated with longer stride length and high speed, which can be a good start for running [8].

Given that the selected start in this study was long, it can be seen that the difference between the two types of the start may be due to the preference of runners to use a short one. Kinematic variables such as knee angle, ankle angle, and shoulder rotation angle have critical importance in sprinting and have an apparent effect on sprint running performance [24]. In contrast, some researchers did not find significant relationships between knee, pelvic, and ankle angles with start performance in sprinting [25].

Researchers suggested the strongest and fastest runners have a fast speed when leaving the start board due to the acute angle of the lower limb joints in a position on the start board, which allows us to have a broader range of joint extensions [26]. This study's selected start had a 100-degree angle in the back knee, which reduced the thigh angle in both legs due to its position. At first, the difference was only $40 \%$, which could be due to this issue. Hunter et al. [27] stated an excellent start in a sprint can increase the horizon- 
tal forces when leaving the starting board and increase the horizontal forces in the following steps.

\section{Ethical Considerations}

Compliance with ethical guidelines

This study was approved by the Ethics Committee of the Kinesiology Research Center, Kharazmi University. (Code:1000/1007).

Funding

The article was extracted from the MSc. thesis of the first author, at Department of Sport Biomechanics, Faculty of Physical Education and Sports Science, Kharazmi University.

Authors' contributions

All authors equally contributed to preparing this article.

Conflicts of interest

The interest of conflict no declared authors. 


\title{
مقايسه التوى هماهنكَى مفاصل اندام تحتانى در استارت دووميدانى اختيارى و منتخب دوندكان نخبه زن
}

\author{
"زهرا بهادرى' هـ، معين كوهستانى' هـ، حيدر صادقى' \\ I. كروه بيومكانيك ورزشى، دانشكده تربيت بدنى و علوم ورزشى، دانشكاه خوارزمى، تهران، ايران.

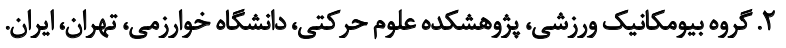

\begin{abstract}
25s

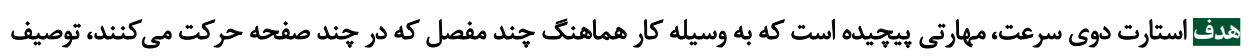

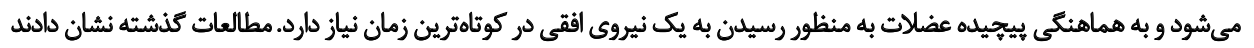

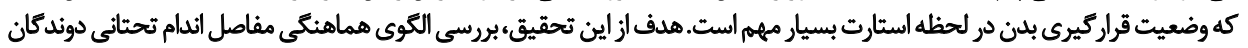
نخبه زن در استارت دوى سرعت بوديت

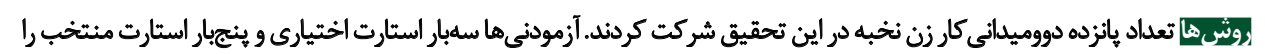

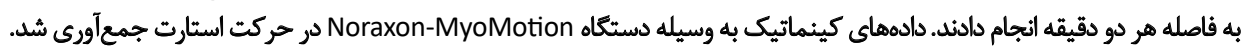

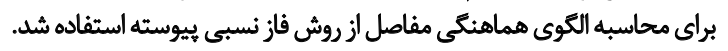

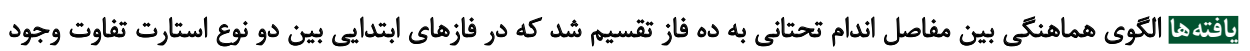

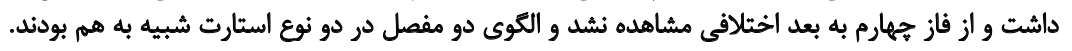

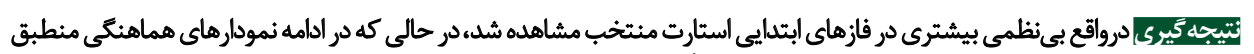

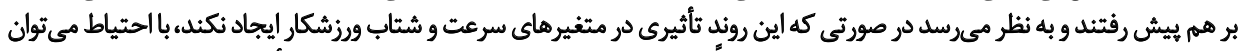

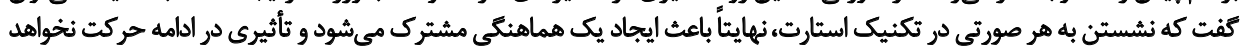

نتايج اين مطالعات نشان مىدهد كه يك جزء ضرورى

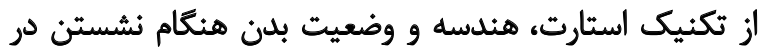

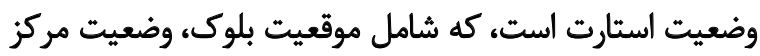

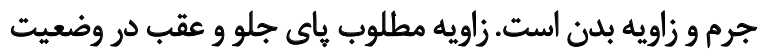

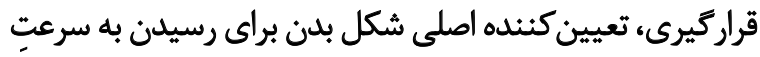

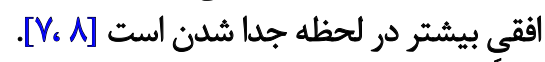

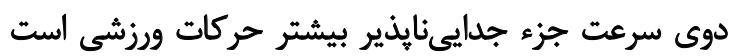

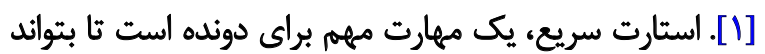

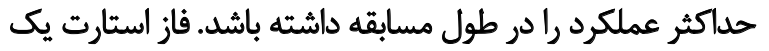

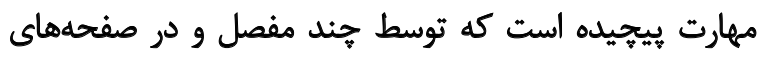

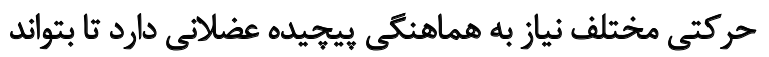

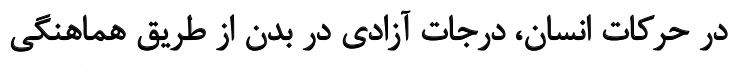

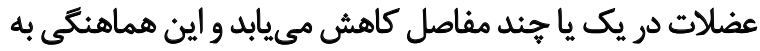

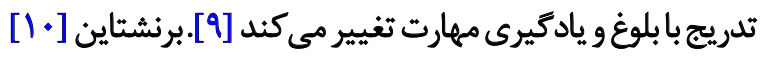

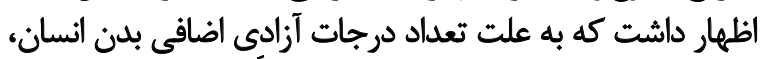

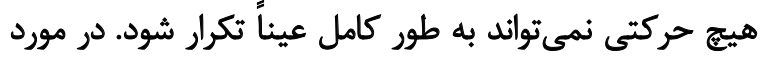

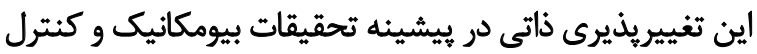

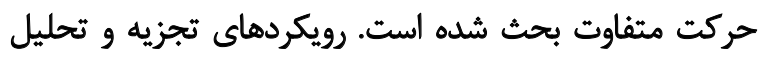

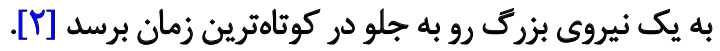
كسب شتاب مؤثر در بخش اول مسابقه دوى سرعت تحت

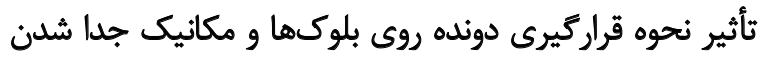

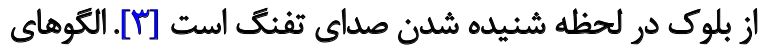

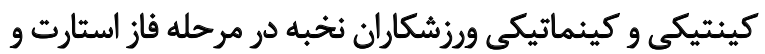

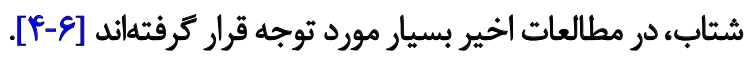


تحتانى جسبانده شد [ه إ] فركانس دستكاه براى ضبط حركات

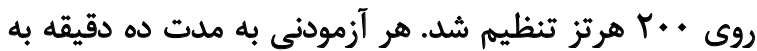

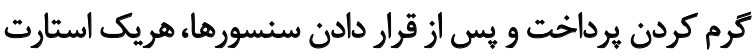

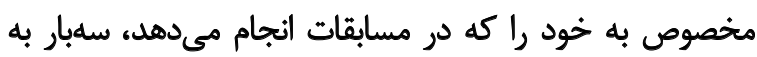

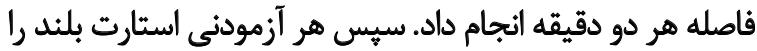

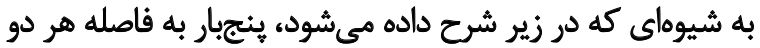
دقيقه انجام داد.

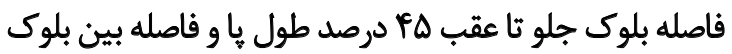

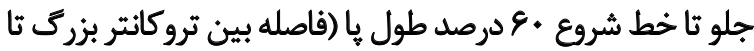

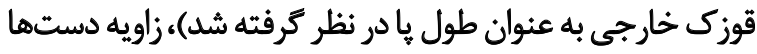

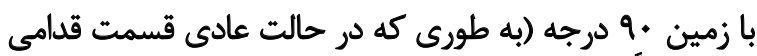

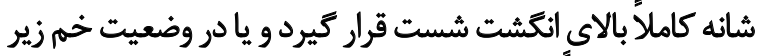

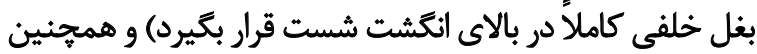

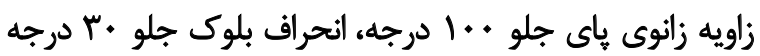

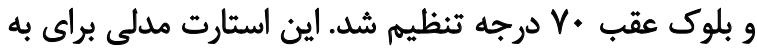

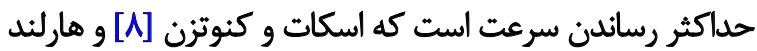
و استيل [19] آن را ييشنهاد دادند.

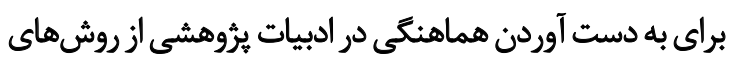

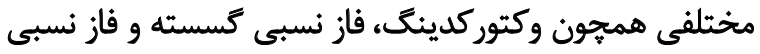

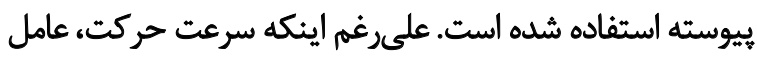

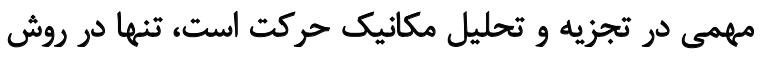

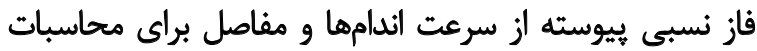

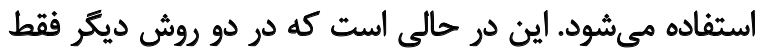

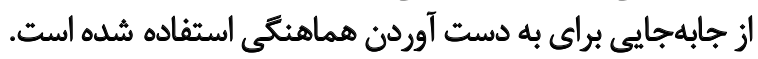

به دليل اهميت سرعت حركت در مهارت استارت، در تحقيق

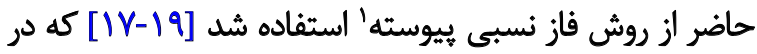

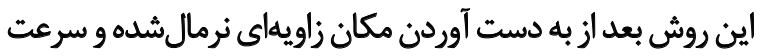

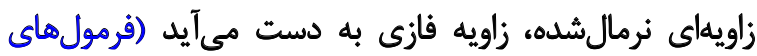

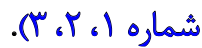

$$
\begin{gathered}
\text { Oinorm }=\left(\frac{2 \times[\theta t-\min (\theta t)]}{\max (\theta t)-\min (\theta t)}\right)-1 \\
\text { winorm }=\left(\frac{\omega i}{\max \{|\omega i|\}}\right) \\
\varnothing=\tan ^{-1}\left(\frac{\omega i}{\theta i}\right) \quad i=12000 n
\end{gathered}
$$

در روابط بالا هø زاويهى فازى مفصل است. در رابطه سه با

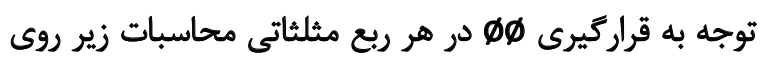

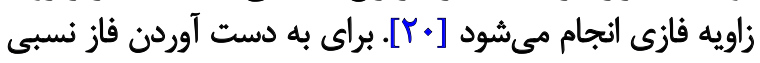

1. Continuous Relative Phase
حركتى مرسوم اغلب تغييريذيرى حركتى درون فرد را ناديده

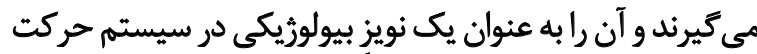

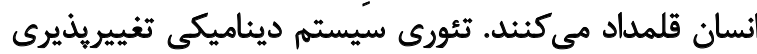

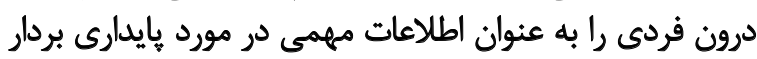

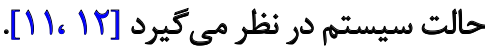

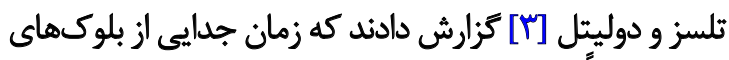

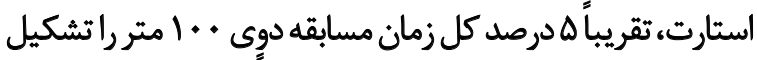

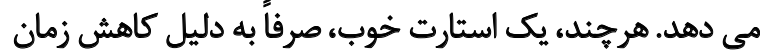

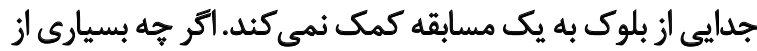

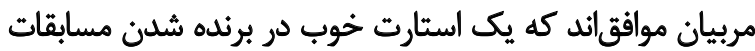

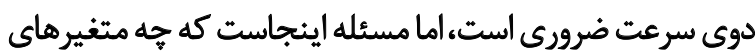
بيومكانيكى در تكنيك استارت نقش دارئ دارند و كداميك ازئا اينها بايد اصلاح شود تا شاهد بهبود عملكرد دوندهنها باشيم.

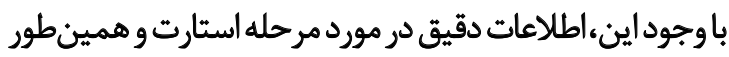

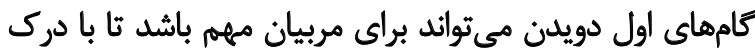

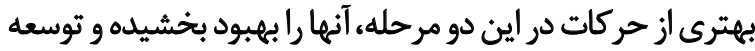

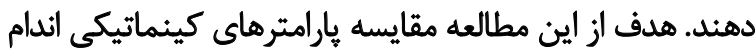

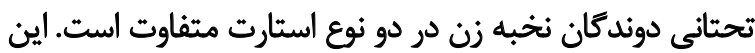
تجزيه و تحليل، هماهنكي بين مفصل دهاي اندام تحتانى ران دادر فاز

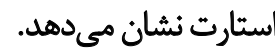

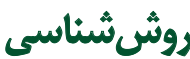

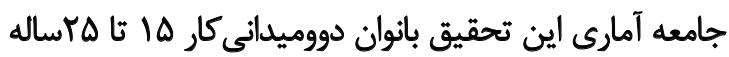

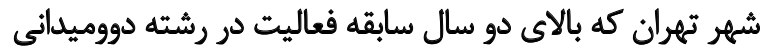

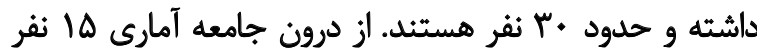

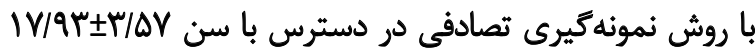

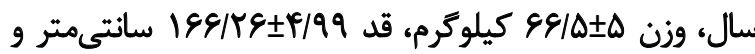

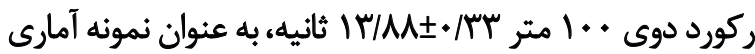

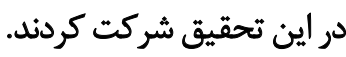

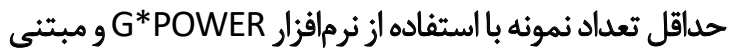

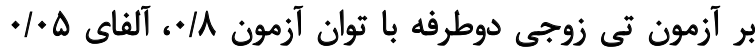

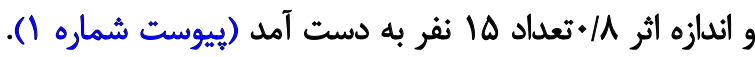

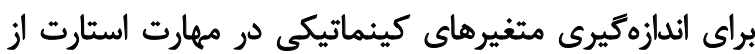
دستكاه Noraxon-MyoMotion ساخت كشور آمريكا استفاده

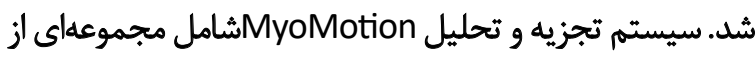

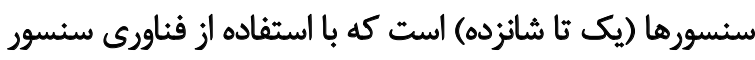

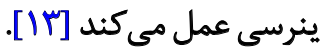

دادههاى جمعآورى شده توسط دستكاه MyoMotion نيز

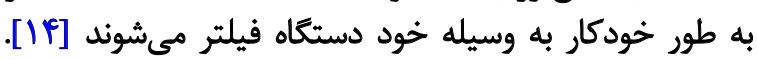

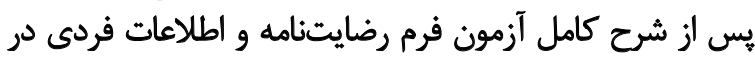

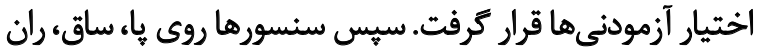

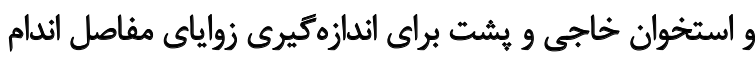


$\omega$

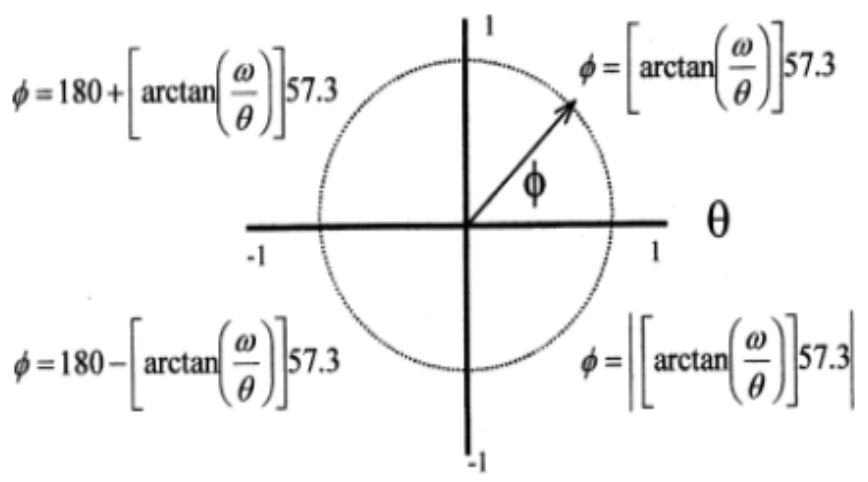

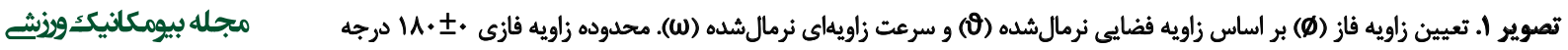

اختلاف بين دو استارت معنادار شده با علامت در داخل نمودار

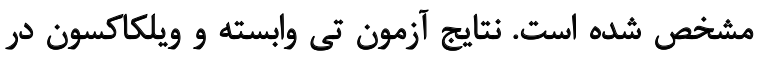

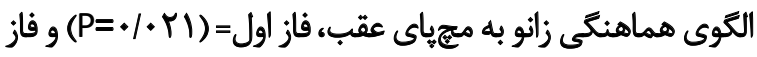

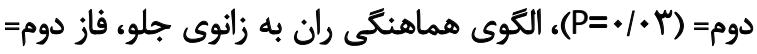

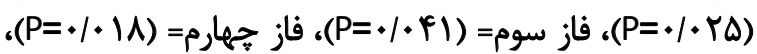

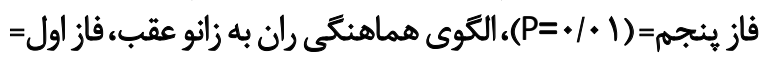

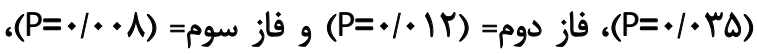

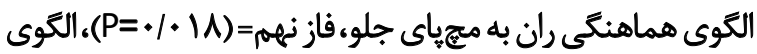

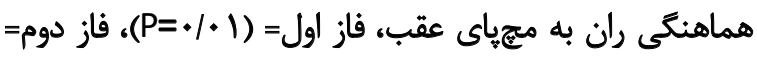

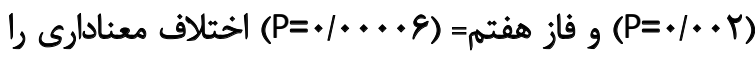
نشان داد و در بقيه موارد اختلاف معنادارى مشاهده نشد. همان طور كه در تصويرهاي شمارها ، ب و Y مشاهده مي كنيد،

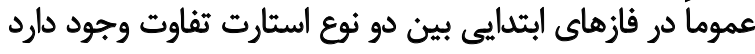

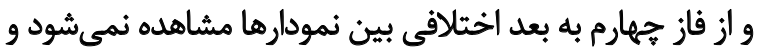

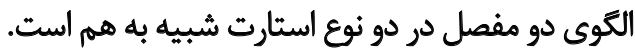

ث

مطالعه حاضر نشان داد كه التوى هماهنگیى اندام تحتانى در فازهاى ابتدايى بين دو نوع استارت تثاوت معنادارى داش داشت، اما

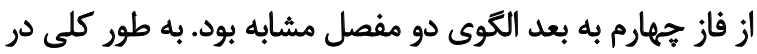

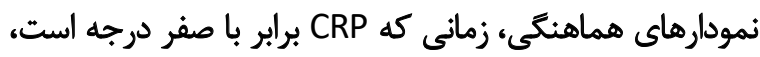

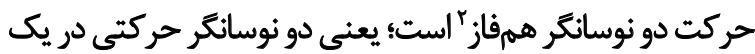

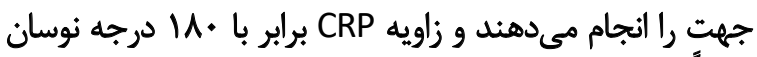

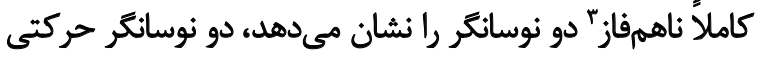

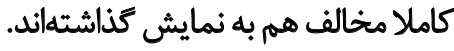

هر زاويه CRP بين صفر ثا • 11 درجه نشاندهنده نوسانات

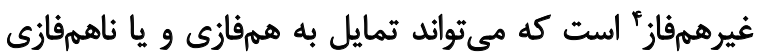

2. In-phase

3. Anti-phase

4. Out of phase
ييوسته زاويه فازى مفاصل تحتانى از زاويه فازى مفاصل فوقانى كم شد (فرمول شماره f).

$\theta$ relative ashehp $=\varnothing$ distal joint $-\varnothing$ proximal joint . . تمامي مراحل مذكور براي اندام يا مفصلى كه با اندام يا مفصل

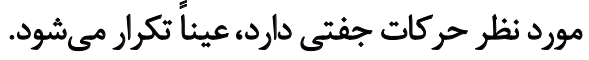

براى آناليز دادهها در مهارت استارت، تمامى دادههاي هماهنكى إنى

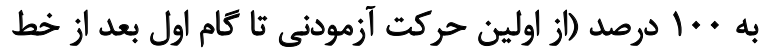

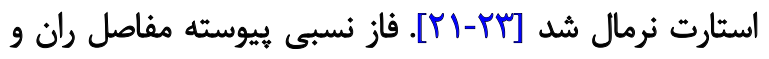

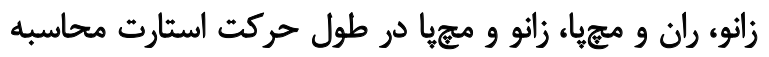
[IV-19] شيد

رايجترين مقاديرى كه از دادههاي CRP مشتق مي شود، شامل

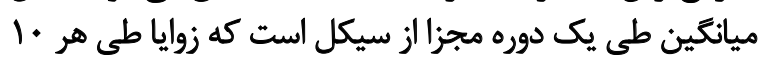

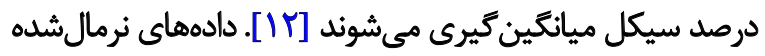

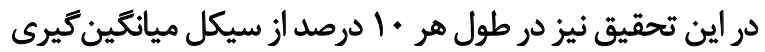
شدند.

براى تحليل آمارى، از آمار توصيفي ميائغين و انحراف استاندارد

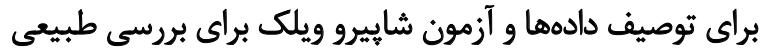

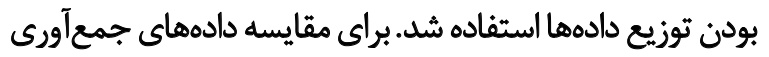

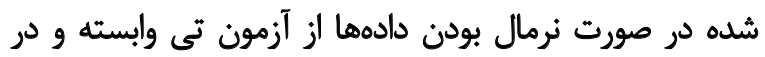

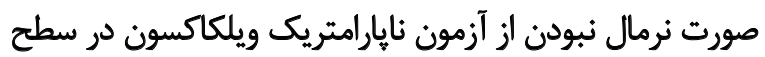

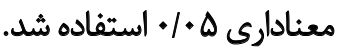

ثنإنج

در مواردى كه آزمون شاييرو ويلك توزيع طبيعى دادهها

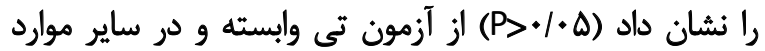

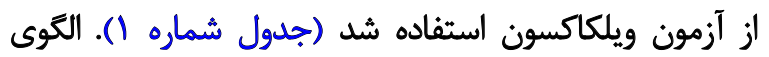

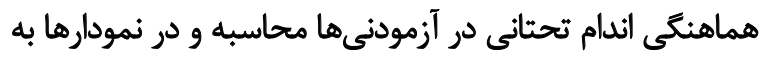

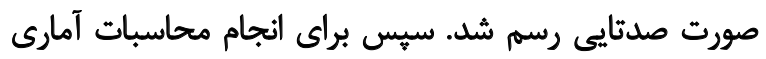

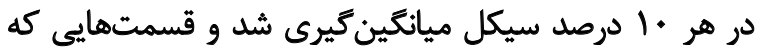



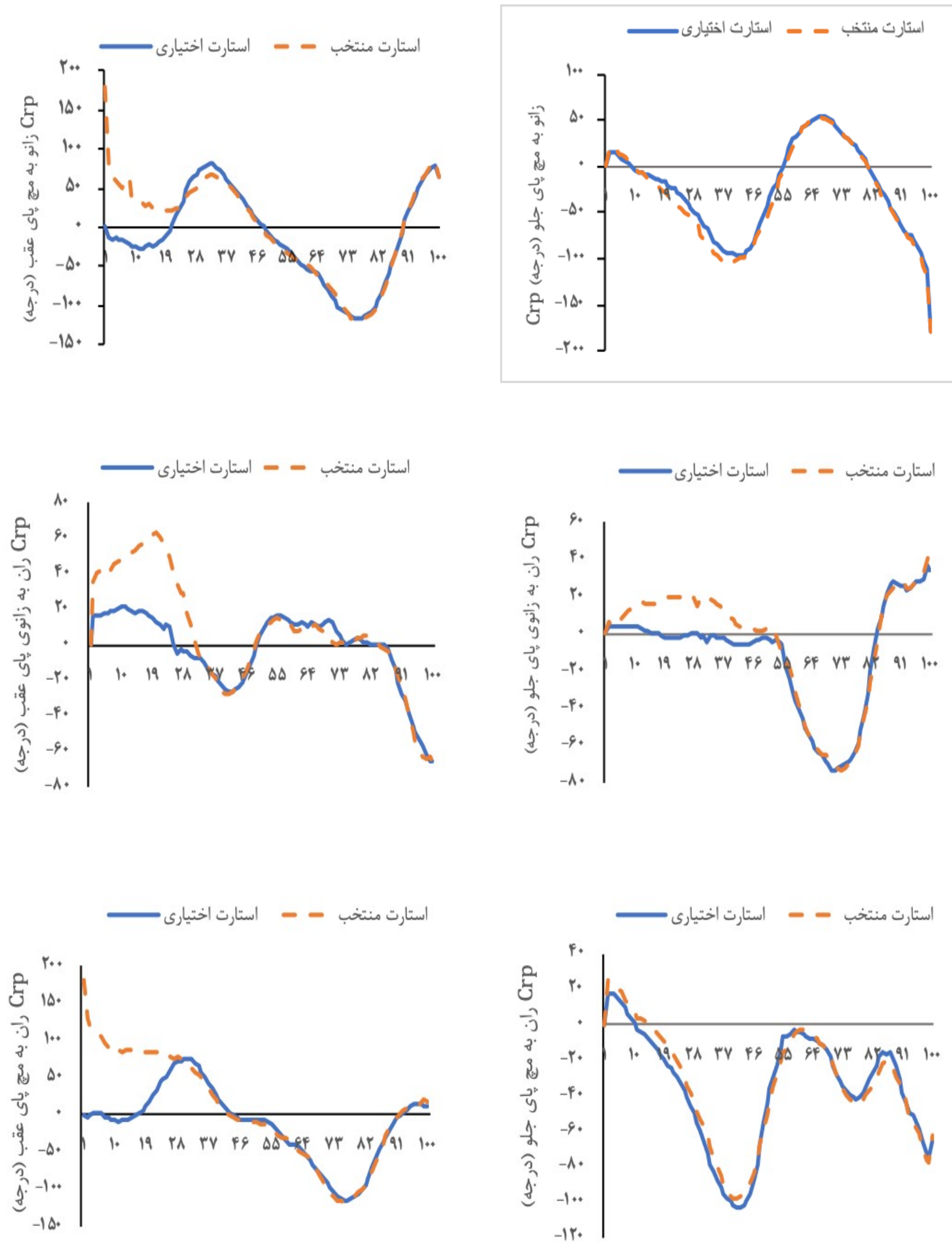

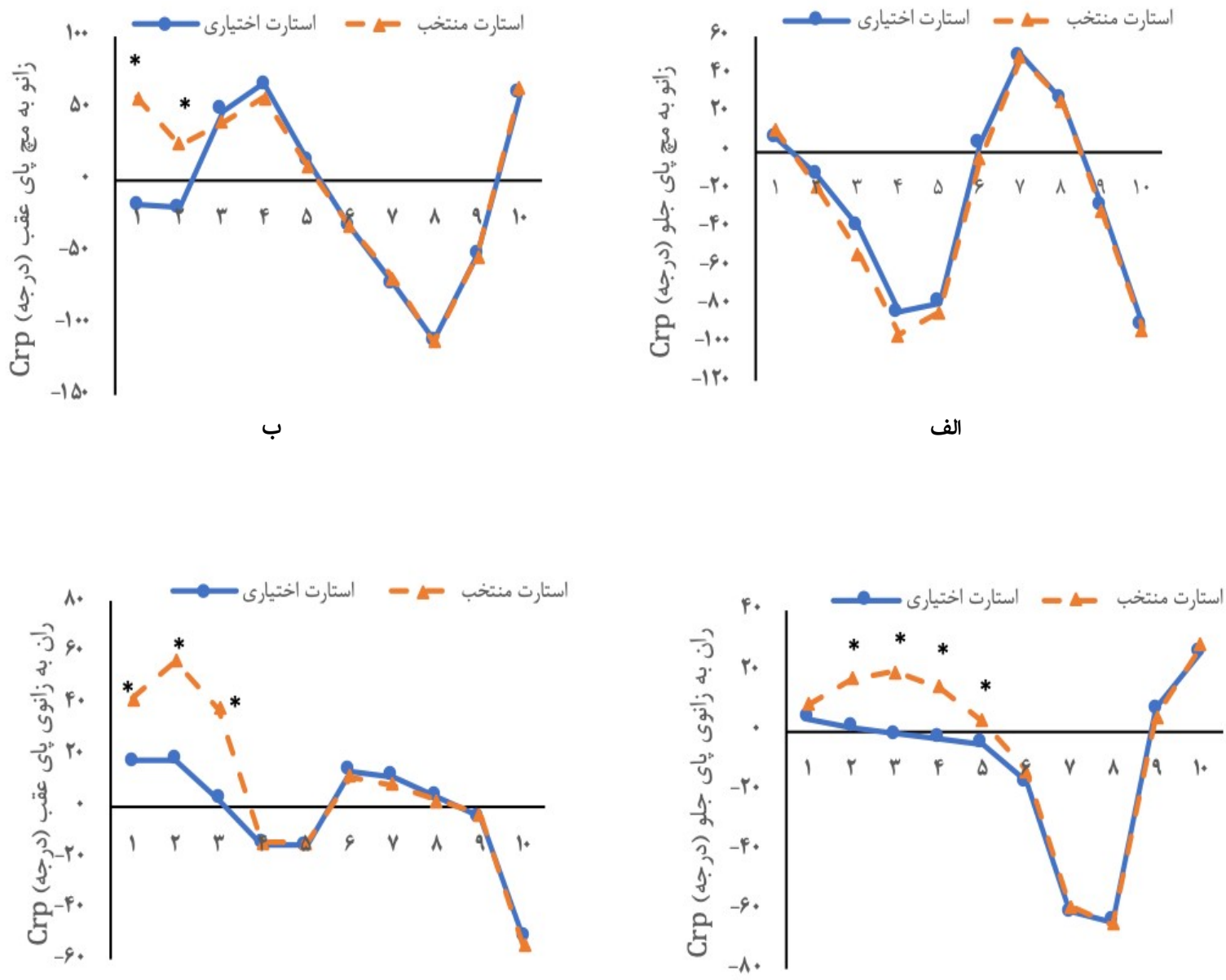

ج
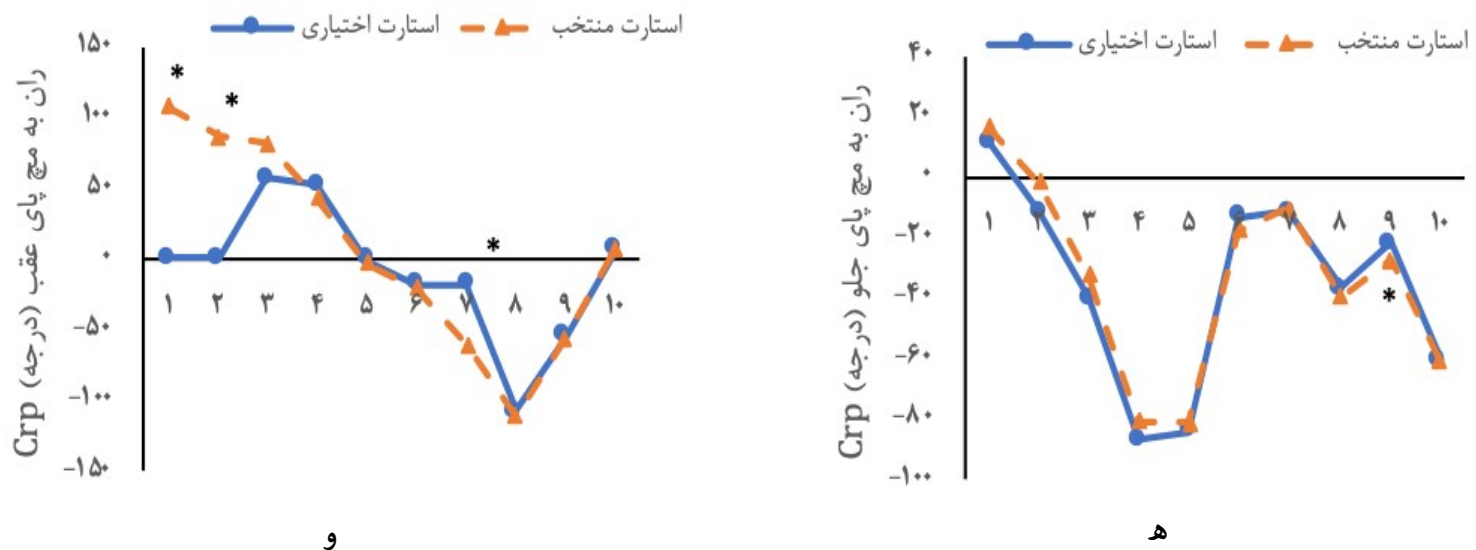

مجله بيومكانيك ونشثـ

تصوير بّ. نمودار الكوى هماهنكي مفاصل اندام تحتانى در ده فاز

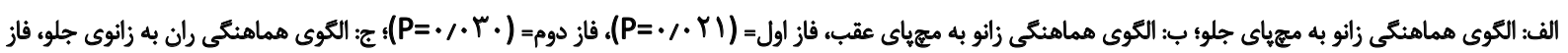

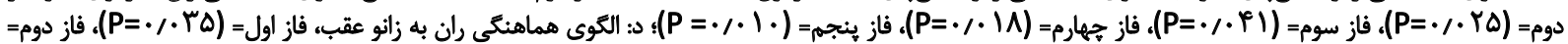

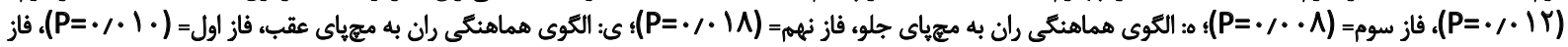

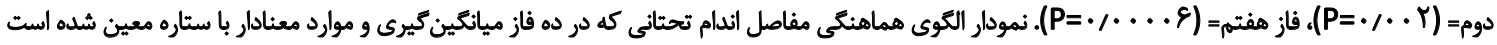


جدول ا. نتايج آزمون شاييرو ويلك توزيع اختلاف متغيرها

\begin{tabular}{|c|c|c|c|c|c|c|c|c|c|}
\hline مثغير & $\mathbf{P}$ & متغير & $\mathbf{P}$ & متغير & $\mathbf{P}$ & متغير & $\mathbf{P}$ & مثغير & $\mathbf{P}$ \\
\hline 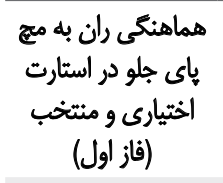 & 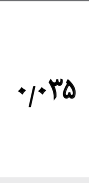 & 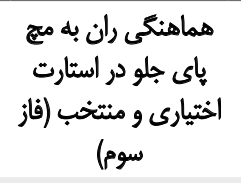 & & 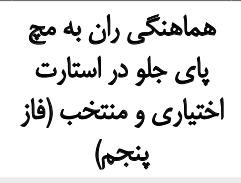 & tpor & 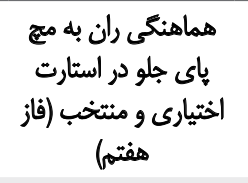 & $<+. .+1$ & 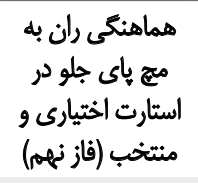 & $<\cdot \%+1$ \\
\hline 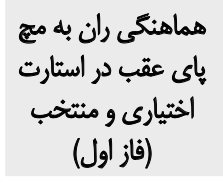 & $\cdot \% \cdot 1 \Delta$ & 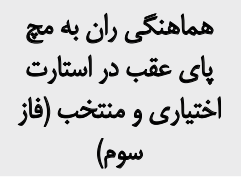 & $\cdot \beta+9$ & 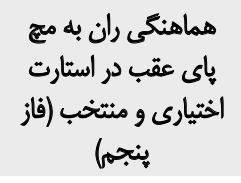 & •APA & 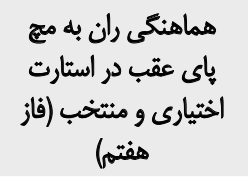 & .1 .1 & 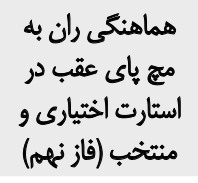 & .198 \\
\hline 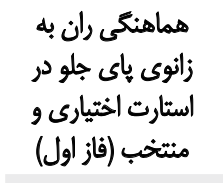 & $.1 . .1$ & 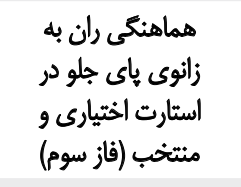 & r & 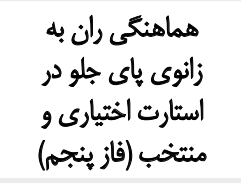 & ${ }^{\prime . . . r}$ & 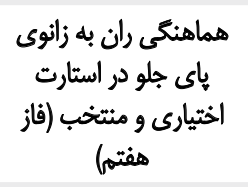 & $<\cdot \mu \cdot \mid$ & 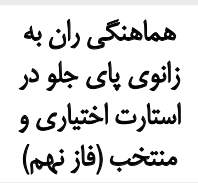 & .918 \\
\hline زانوى بأى عقب دان بهر & $\bullet \bullet \wedge 1$ & 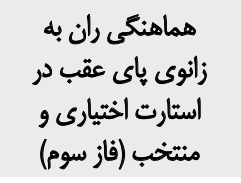 & $\cdot \pi+8$ & زانوى يائى عقب دران بـ & $\%$ Y & 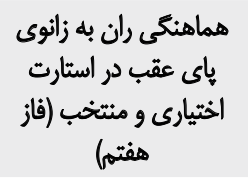 & $\pi \cdot 1$ & 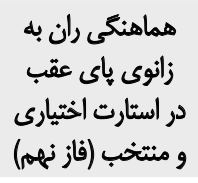 & זעקו. \\
\hline 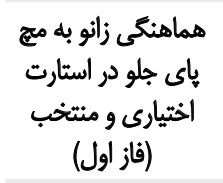 & .199 & 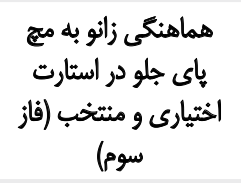 &., $1 r$ & 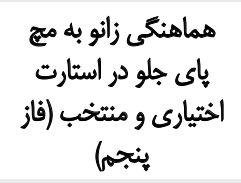 & - p*Aq & 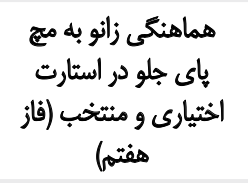 & $\cdot \% \cdot \gamma$ & 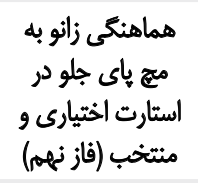 & 091. \\
\hline 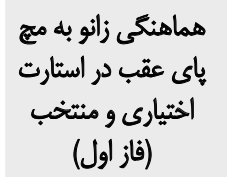 & $* 1 .+4$ & 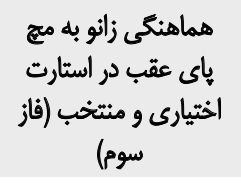 & . $\mu$. & 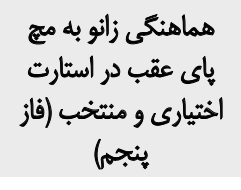 & - llef & 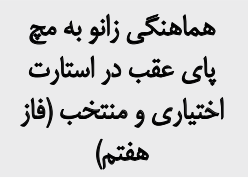 & r & 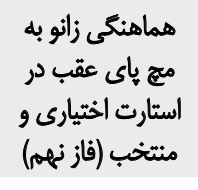 & ס ס \\
\hline 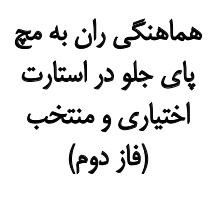 & $+1+49$ & 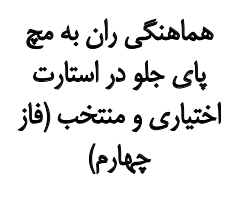 & ${ }^{*}$ Mr & اخثيارى ولو مثتخب استارت (فاز & +1.94 & 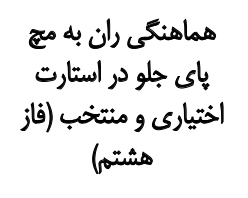 & $\cdot \pi+$ & 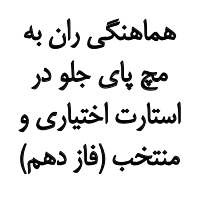 & \\
\hline 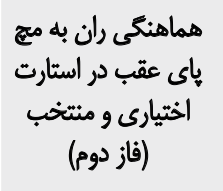 &.$/$ Af & 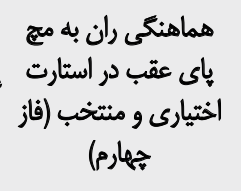 & pres & 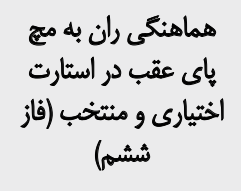 & 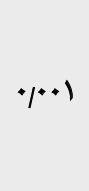 & 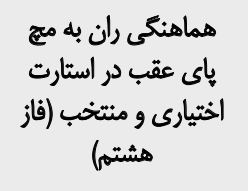 & . $\Gamma \Delta A$ & 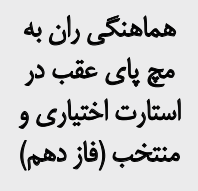 & سזקק. \\
\hline 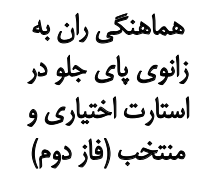 & $\%$ & 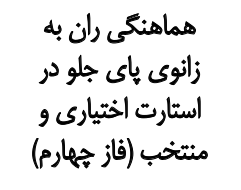 & $\cdot \Delta \gamma^{\prime \prime}$ & 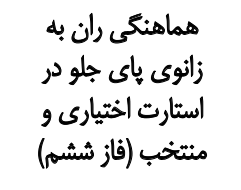 & - MAq & 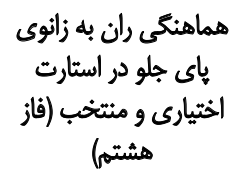 & $0 N \cdot 8$ & 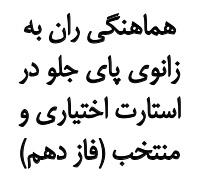 & . AIr \\
\hline زانوى باهنى عقب دان ديه & +11.8 & 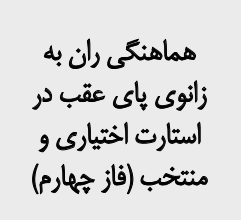 & $\langle+|\cdot+|$ & 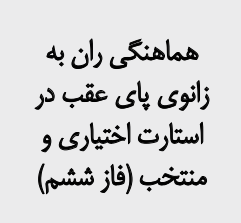 & . & 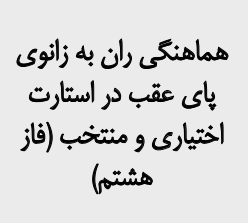 & هזr & 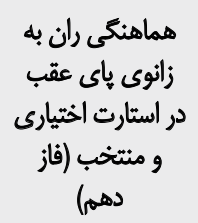 & $+\mid r \lambda$ \\
\hline
\end{tabular}




\begin{tabular}{|c|c|c|c|c|c|c|c|c|c|}
\hline متغير & $\mathbf{P}$ & متغير & $\mathbf{P}$ & مثتير & $\mathbf{P}$ & متغير & $\mathbf{P}$ & متغير & $\mathbf{P}$ \\
\hline 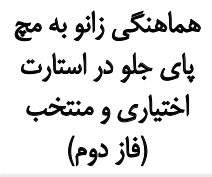 & 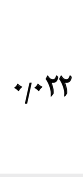 & 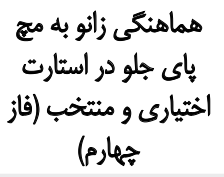 & $\cdot 1 \cdot \gamma$ & 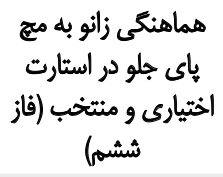 & - jeva & 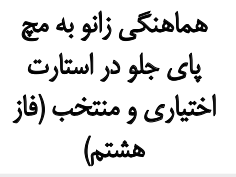 & / pr. & هماهنكي زانو بيه & $\%$. rq \\
\hline 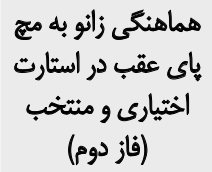 & "זון & 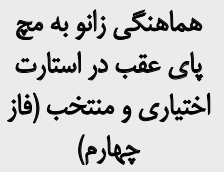 & . pr. & 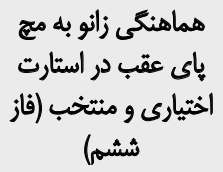 & $\cdot r+\Delta$ & 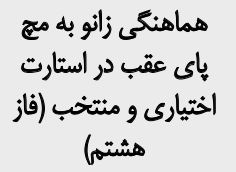 & $\cdot \Delta \Delta \Delta \Delta$ & 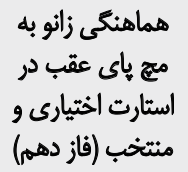 & $\cdot \mid \Delta \Delta \Delta \Delta$ \\
\hline
\end{tabular}

مجله بيومكانيك وزنش

حالت همفازى حركت رآعاز كردهاند كه در استارت اختيارى اين

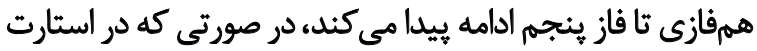

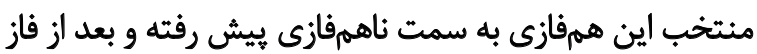

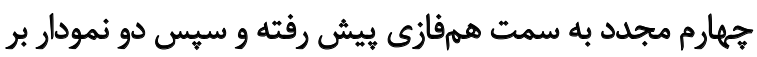
هم منطبق شدهاند.

هماهنكى ران به مج خاى جلو: در استارت اختيارى حركت به

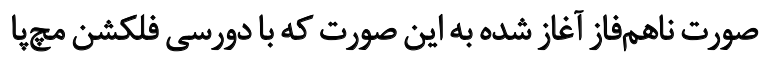

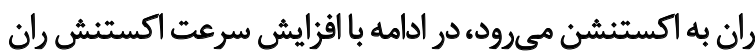

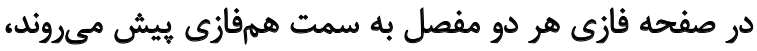

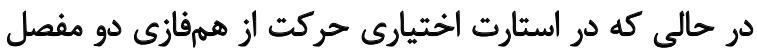

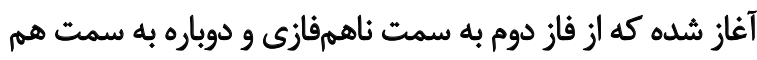

فازى ميرود.

دو نمودار از فاز جهارم به بعد با هم منطبق مي دشوند. از فاز

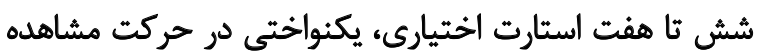

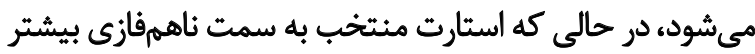

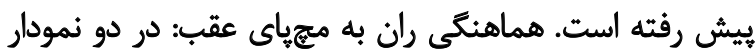

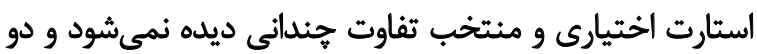
نمودار بر هم منطبق هستئند

اسكات بيان داشت كه استارت كوتاه، سرعت بالاتر و استارت

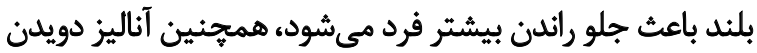

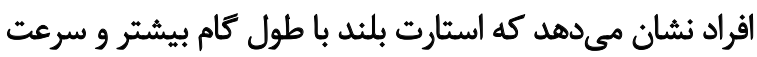

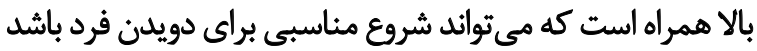

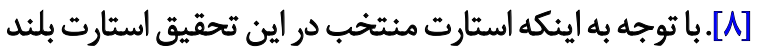

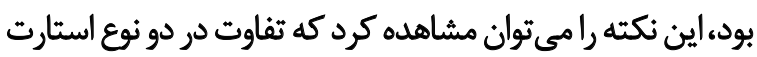

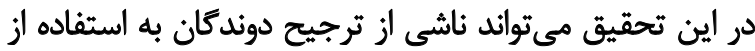

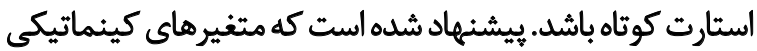

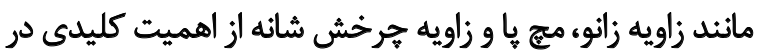

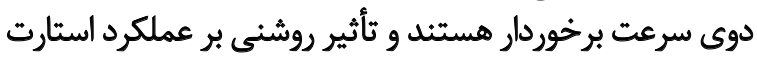

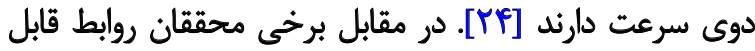

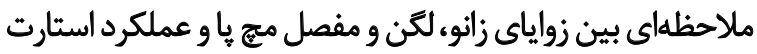

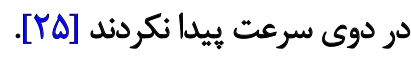

داشته باشد. مقادير CRP مثبت نشان مي دهد كه اندام ديستال

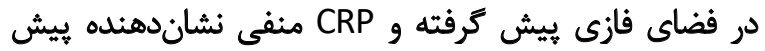

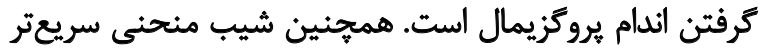

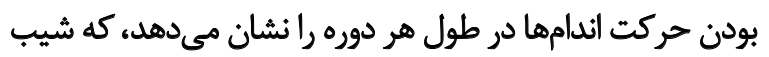

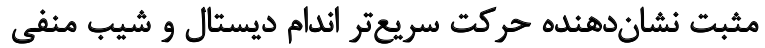

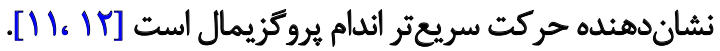

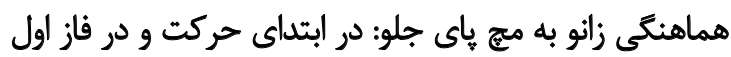

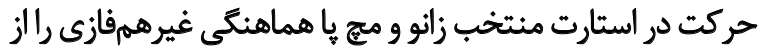

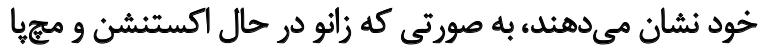

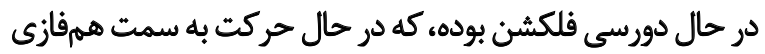

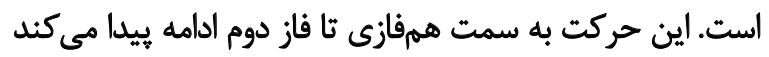

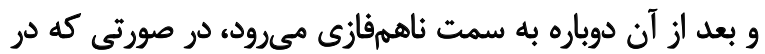

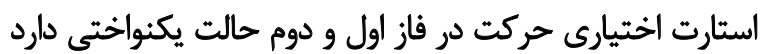

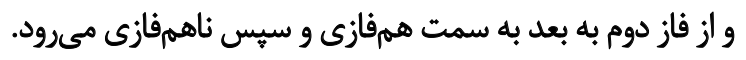
در استارت منتخب، در ابتداى حركت زانو حركت سريعترى

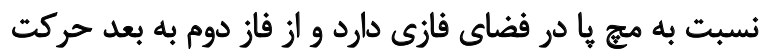

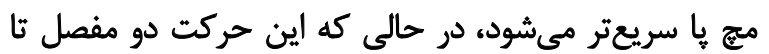

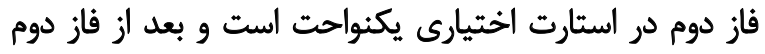

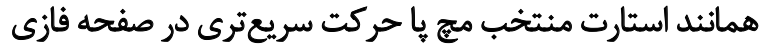

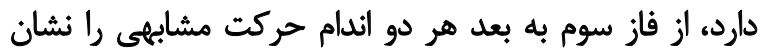

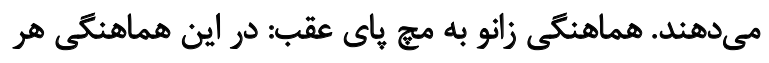

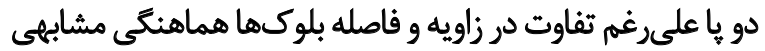
رانشان مي دهئد.

هماهنكى ران به زانوى جلو: در استارت منتخب دو ماند مفصل

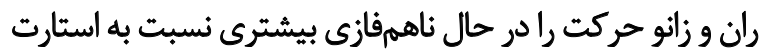

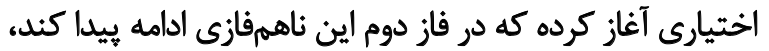

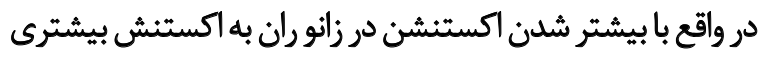

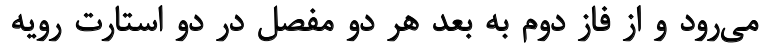

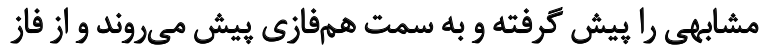
جهار به بعد دو نمودار بر هم منطبق ونه شدند.

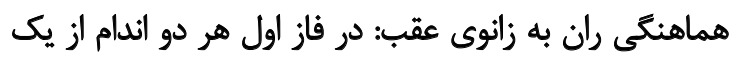




\section{خوارزمى استخراج شده است. \\ مشاركت نويسند مكان}

تمامى نويسندكان در انجام و نكارش مطالعه حاضر، به ميزان

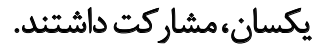

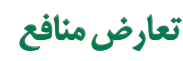

بنابر اظهار نويسندكان،اين مقاله هيج كونه تعارض منافع ندارد.

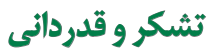

بدينوسيله نويسندكان اين مقاله از تمامى كساني كه در انجام

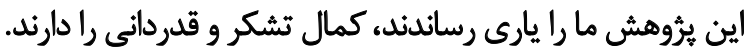

محققان مطرح كردند كه قوىترين و سريعترين دوندكان

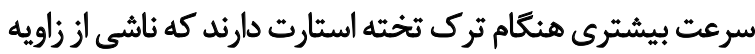

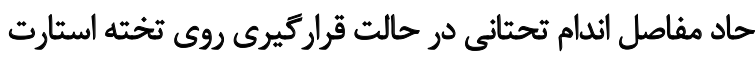

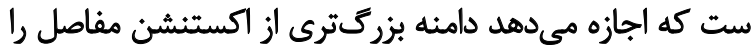

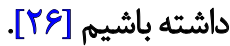

با توجه به اينكه در اين تحقيق در استارت نوع منتخب زاويه

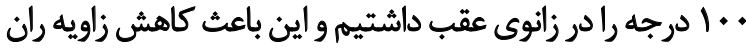

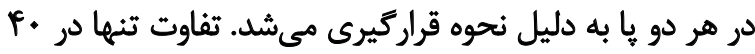

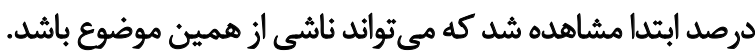

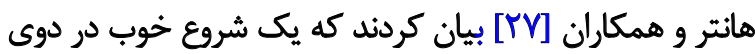

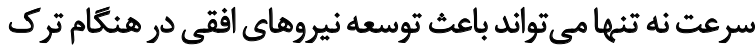

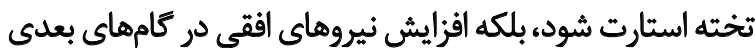

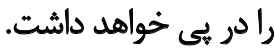

\section{نتيجه كيرى نهايي}

با توجه به اينكه تفاوت در دو نمودار تنها در فازهاى ابتدايى

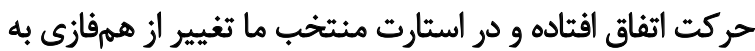

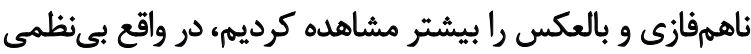

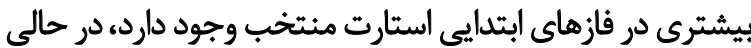

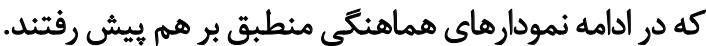

افراد نوع حركت در استارت منتخب (استارت منتخب در اين

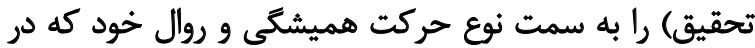

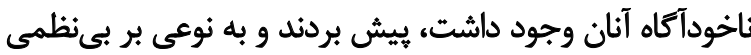

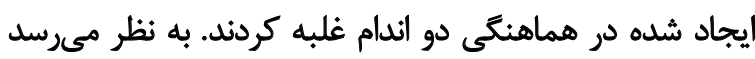

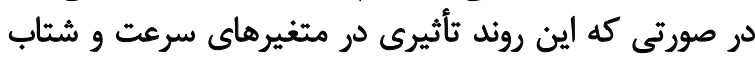

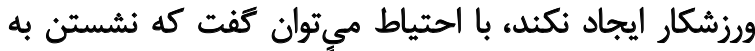

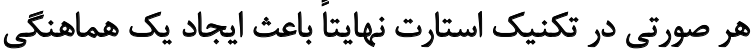

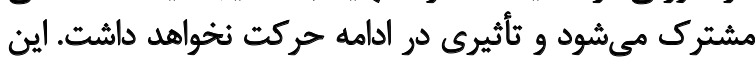

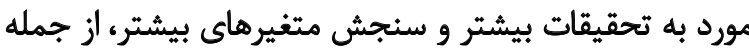

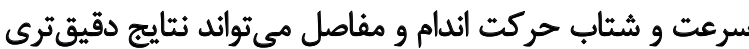

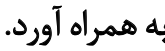

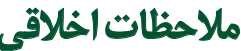
ييروى از اصول اخلاق يثوهش - ميش

شايان ذكر است تمامى آزمودنيها فرم رضايتنامه كتبى

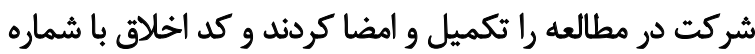

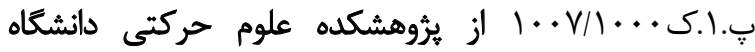
خوارزمى توسط يثروهشكر اخذ شد.

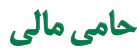

اين مقاله از باياننامه كارشناسى ارشد نويسنده اول در كروه بيومكانيك ورزشى دانشكده تربيت بدنى و علوم ورزشى ارشى دانشكاه 


\section{References}

[1] Cronin JB, Hansen KT. Strength and power predictors of sports speed. J Strength Cond Res. 2005; 19(2): 349-57. [DOI:10.1519/00124278200505000-00019] [PMID]

[2] Harland MJ, Andrews MH, Steele JR. Instrumented start blocks: A quantitative coaching aid. ISBS-Conference Proceedings Archive; 1995. https://ojs.ub.uni-konstanz.de/cpa/article/view/2952

[3] Tellez T, Doolittle D. Sprinting from start to finish. Track Tech. 1984 88:2802-5. https://scholar.google.com/scholar?hl=nl\&as_sdt=0\%2C $5 \& q=$ Sprinting+from+start+to+finish.\&btnG $=$

[4] Bezodis NE, Salo Al, Trewartha G. Choice of sprint start performance measure affects the performance-based ranking within a group of sprinters: Which is the most appropriate measure? Sports Biomech. 2010; 9(4):258-69. [DOI:10.1080/14763141.2010.538713] [PMID]

[5] Čoh M, Tomažin K. Kinematic analysis of the sprint start and acceleration from the blocks. New Stud in Athletics. 2006; 21(3):23-33. https://www.researchgate.net/publication/298645089_Kinematic analysis_of_the_sprint_start_and_acceleration_from_the_blocks

[6] Slawinski J, Bonnefoy A, Levêque JM, Ontanon G, Riquet A, Dumas $R$, et al. Kinematic and kinetic comparisons of elite and well-trained sprinters during sprint start. J Strength Cond Res. 2010; 24(4):896905. [DOI:10.1519/JSC.0b013e3181ad3448] [PMID]

[7] Mero A, Kuitunen S, Harland M, Kyröläinen H, Komi PV. Effects of muscletendon length on joint moment and power during sprint starts. J Sports Sci. 2006; 24(2):165-73. [DOI:10.1080/02640410500131753] [PMID]

[8] Schot PK, Knutzen KM. A biomechanical analysis of four sprint start positions. Res Q Exerc Sport. 1992; 63(2):137-47. [DOI:10.1080/027 01367.1992.10607573] [PMID]

[9] Thelen E. Motor development: A new synthesis. Am Psychol. 1995 50(2):79-95. [DOI:10.1037/0003-066X.50.2.79]

[10] Bernstein N. The co-ordination and regulation of movements. $1^{\text {st }}$ English ed. London: Pergamon; 1967.

[11] Stergiou N. Innovative Analyses of Human Movement. Champaign IL,: Human Kinetics; 2004. https://www.amazon.com/InnovativeAnalyses-Movement-Nicholas-Stergiou/dp/0736044671

[12] Robertson GD, Caldwell GE, Hamill J, Kamen G. Research methods in biomechanics, $2^{\text {nd }}$ edition. Champaign, IL,: Human Kinetics; 2013. [DOI:10.5040/9781492595809]

[13] Wolfgang T. Biomechanical quantification of the dynamic knee valgus using inertial sensor system myomotion. Gießen: Justus; 2016

[14] Balasubramanian S. Comparison of angle measurements between vicon and myomotion system. Arizona: Arizona State University. 2013. https://www.semanticscholar.org/paper/COMPARISONOF-ANGLE-MEASUREMENTS-BETWEEN-VICON-AND/1641d6ece74c8e7e9e5f06595bad7b49540bc53a

[15] Noraxon. MyoMotion System User Guide [Intenet]. 2014 [Updated 2014]. Available from: https://www.noraxon.com/noraxon-download/ myomotion-system-user-manual/

[16] Harland M, Steele JR. Biomechanics of the sprint start. J Sports Medicine. 1997; 23(1):11-20. [DOI:10.2165/00007256-19972301000002] [PMID]

[17] Khezri D, Eslami M, Yaserifar M. [The effect of variation of shoe sole stiffness on coordination pattern and it's variability in tars-metatarsal and forefoot-hallux joints during stance phase of running (Per- sian)]. International Conference on Physical Education and Sports. 19-20 March 2016; Tehran: Kharazmi University. https://www.sid.ir/ $\mathrm{Fa} /$ Seminar/ViewPaper.aspx?ID=26874

[18] Silvernail JF, Boyer K, Rohr E, Brüggemann GP, Hamill J. Running mechanics and variability with aging. J Med Sci Sports Exerc. 2015; 47(10):2175-80. [DOI:10.1249/MSS.0000000000000633] [PMID]

[19] Wheat JS, Glazier PS. Measuring coordination and variability in coordination. In: Davids K, Bennett S, Newel K, editors. Movement System Variability. Champaign: Human Kinetics; 2005 [DOI:10.5040/9781492596851.ch-009]

[20] Hamill J, van Emmerik RE, Heiderscheit BC, Li L. A dynamical systems approach to lower extremity running injuries. Clin Biomech (Bristol, Avon). 1999; 14(5):297-308. [DOI:10.1016/S0268-0033(98)90092-4]

[21] Milanese C, Bertucco M, Zancanaro C. The effects of three different rear knee angles on kinematics in the sprint start. J Biology of Sport. 2014; 31(3):209-15. [DOI:10.5604/20831862.1111848] [PMID] [PMCID]

[22] Ciacci S, Merni F, Bartolomei S, Di Michele R. Sprint start kinematics during competition in elite and world-class male and female sprinters. J Sports Sci. 2017; 35(13):1270-8. [DOI:10.1080/02640414.2016 .1221519] [PMID]

[23] Chen Y, Wu KY, Tsai YJ, Yang WT, Chang JH. The kinematic differences of three types of crouched position during sprint start. J Mech Med Biol. 2016; 16(7):1650099. [DOI:10.1142/S0219519416500998]

[24] Ansari NW, Paul Y, Sharma K. Kinematic analysis of competitive sprinting: Biomechanics. Afr J Phys Health Educ Recreat Dance. 2012; 18(4):662-71. https://www.ajol.info/index.php/ajpherd/article/view/83832

[25] Chakravarty R. The relationship of selected kinematical variables of the performance of runners in sprint start. Int J Sports Sci Fit. 2011; 1(1):60-7. https://web.a.ebscohost.com/abstract?direct=true\&profile= ehost\&scope=site\&authtype=crawler\&jrnl

[26] Maulder PS, Bradshaw EJ, Keogh J. Jump kinetic determinants of sprint acceleration performance from starting blocks in male sprinters. J Sports Sci Med. 2006; 5(2):359-66. [PMID] [PMCID]

[27] Hunter JP, Marshall RN, McNair PJ. Interaction of step length and step rate during sprint running. J Med Sci Sports Exerc. 2004 36(2):261-71. [DOI:10.1249/01.MSS.0000113664.15777.53] [PMID] 


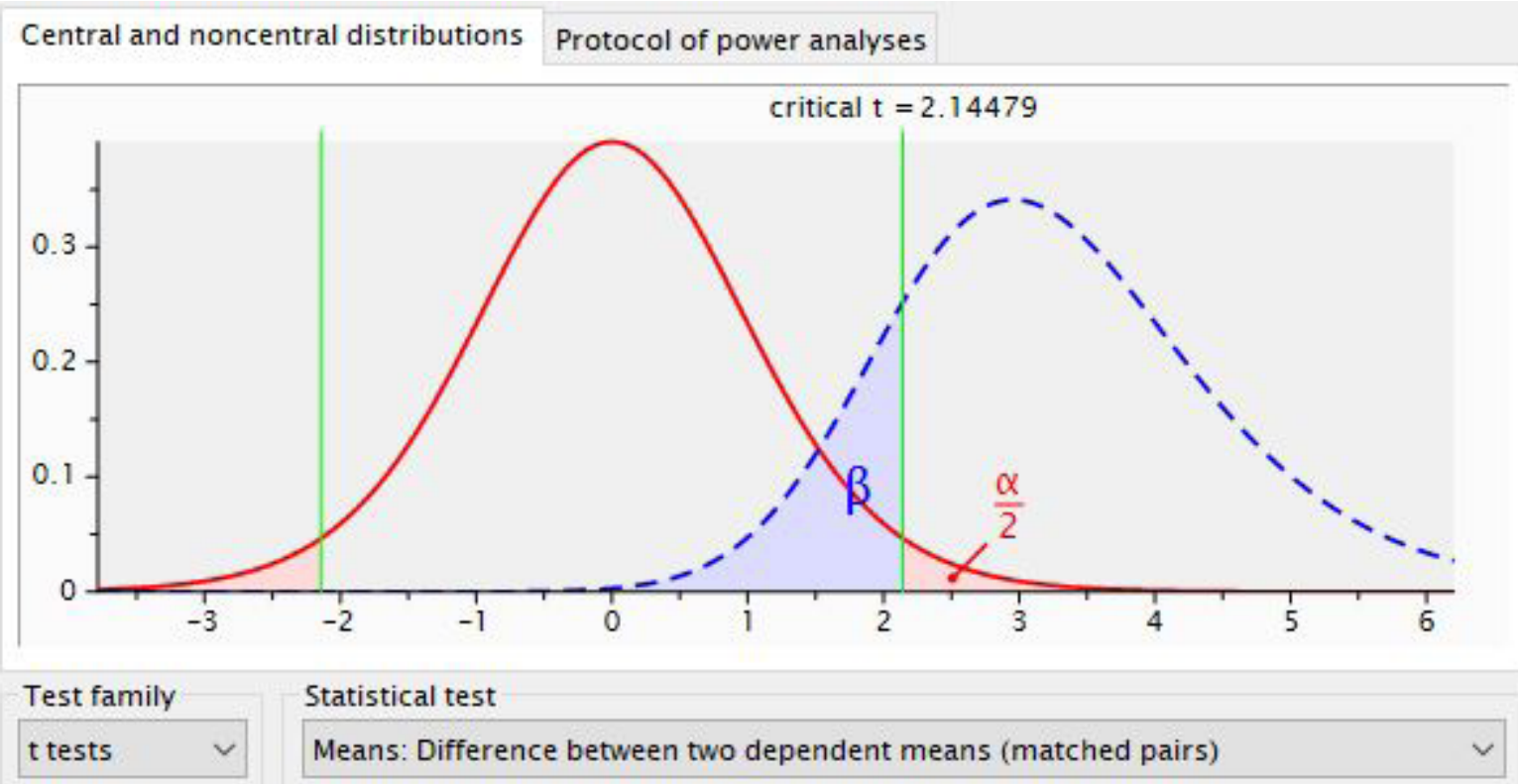

Type of power analysis

A priori: Compute required sample size - given $\alpha$, power, and effect size

Input Parameters

\begin{tabular}{rlr|} 
Tail(s) & Two \\
\cline { 3 - 3 } Determine $=>$ Effect size dz & $\checkmark$ \\
\cline { 2 - 2 } & & 0.8 \\
$\alpha$ err prob & 0.05 \\
& Power (1- $\beta$ err prob) & 0.8 \\
\hline
\end{tabular}

\section{Output Parameters}

Noncentrality parameter $\delta$

Critical t

\begin{tabular}{|r|}
\hline 3.0983867 \\
\hline \hline 2.1447867 \\
\hline
\end{tabular}

\begin{tabular}{r|r} 
Critical t & 2.1447867 \\
\cline { 2 - 2 } Df & 14 \\
\cline { 2 - 2 } Total sample size & 15 \\
\cline { 2 - 2 } Actual power & 0.8213105
\end{tabular}


This Page Intentionally Left Blank 